\title{
Locating Hydrides in Ligand-Protected Copper Nanoclusters by Deep Learning
}

\author{
Song Wang, Tongyu Liu, and De-en Jiang* \\ Department of Chemistry, University of California, Riverside, California 92521, United States \\ *Corresponding author. Email: djiang@ucr.edu
}

Table S1. CNN-predicted most probable hydride sites in $\left[\mathrm{Cu}_{81}(\mathrm{PhS})_{46}\left({ }^{\mathrm{t}} \mathrm{BuNH}_{2}\right)_{10}(\mathrm{H})_{32}\right]^{3+}$ and the site combination in the only surviving isomer (Isomer B) after symmetry constraint $\left(\mathrm{C}_{2}\right)$ : Yes, site has $\mathrm{H}^{-}$; No, site doesn't have $\mathrm{H}^{-}$.

\begin{tabular}{|c|c|c|c|c|c|}
\hline Site Rank & $\begin{array}{c}\text { Probability of H- } \\
\text { occupancy from CNN }\end{array}$ & Isomer B & Site Rank & $\begin{array}{c}\text { Probability of H- } \\
\text { occupancy from CNN }\end{array}$ & Isomer B \\
\hline 1 & $99.97 \%$ & Yes & 19 & $99.25 \%$ & Yes \\
\hline 2 & $99.95 \%$ & Yes & 20 & $99.18 \%$ & Yes \\
\hline 3 & $99.94 \%$ & Yes & 21 & $99.13 \%$ & Yes \\
\hline 4 & $99.90 \%$ & Yes & 22 & $99.12 \%$ & Yes \\
\hline 5 & $99.85 \%$ & Yes & 23 & $98.86 \%$ & Yes \\
\hline 6 & $99.77 \%$ & Yes & 24 & $98.81 \%$ & Yes \\
\hline 7 & $99.76 \%$ & Yes & 25 & $98.72 \%$ & Yes \\
\hline 8 & $99.71 \%$ & Yes & 26 & $98.56 \%$ & Yes \\
\hline 9 & $99.70 \%$ & Yes & 27 & $98.25 \%$ & Yes \\
\hline 10 & $99.70 \%$ & Yes & 28 & $97.83 \%$ & Yes \\
\hline 11 & $99.68 \%$ & Yes & 29 & $97.40 \%$ & Yes \\
\hline 12 & $99.65 \%$ & Yes & 30 & $97.29 \%$ & Yes \\
\hline 13 & $99.61 \%$ & Yes & 31 & $97.07 \%$ & Yes \\
\hline 14 & $99.56 \%$ & Yes & 32 & $96.96 \%$ & No \\
\hline 15 & $99.56 \%$ & Yes & 33 & $95.41 \%$ & Yes \\
\hline 16 & $99.37 \%$ & Yes & 34 & $83.09 \%$ & No \\
\hline 17 & $99.33 \%$ & Yes & 35 & $66.93 \%$ & No \\
\hline 18 & $99.28 \%$ & Yes & 36 & $65.09 \%$ & No \\
\hline
\end{tabular}


The coordinates of our $\mathrm{Cu} 81$ isomer (Isomer $\mathrm{B}$ ): $\left[\mathrm{Cu}_{81}\left(\mathrm{CH}_{3} \mathrm{~S}\right)_{46}\left(\mathrm{CH}_{3} \mathrm{NH}_{2}\right)_{10}(\mathrm{H})_{32}\right]^{3+}$

\begin{tabular}{|c|c|c|c|}
\hline Atom & $X$ & $\mathrm{Y}$ & Z \\
\hline $\mathrm{H}$ & 29.571494 & 10.532926 & 15.170960 \\
\hline $\mathrm{H}$ & 30.120680 & 10.764043 & 16.684860 \\
\hline $\mathrm{H}$ & 28.355983 & 14.191714 & 10.002227 \\
\hline $\mathrm{H}$ & 27.351774 & 12.910723 & 10.117972 \\
\hline $\mathrm{H}$ & 32.188424 & 15.295474 & 16.060403 \\
\hline $\mathrm{H}$ & 31.820524 & 14.591439 & 14.639306 \\
\hline $\mathrm{H}$ & 29.891473 & 24.797493 & 16.716927 \\
\hline $\mathrm{H}$ & 29.864898 & 25.423849 & 15.213547 \\
\hline $\mathrm{H}$ & 29.314572 & 12.797466 & 20.813642 \\
\hline $\mathrm{H}$ & 30.168787 & 14.184148 & 20.785957 \\
\hline $\mathrm{H}$ & 20.735145 & 21.142781 & 15.814241 \\
\hline $\mathrm{H}$ & 18.178707 & 15.153556 & 16.058167 \\
\hline $\mathrm{H}$ & 20.993646 & 16.655417 & 15.991661 \\
\hline $\mathrm{H}$ & 24.036494 & 18.647816 & 13.284707 \\
\hline $\mathrm{H}$ & 21.100235 & 18.987349 & 13.724154 \\
\hline $\mathrm{H}$ & 22.827265 & 21.243959 & 13.721413 \\
\hline $\mathrm{H}$ & 25.803698 & 20.912431 & 14.578472 \\
\hline $\mathrm{H}$ & 20.437271 & 23.038548 & 13.604413 \\
\hline $\mathrm{H}$ & 23.717250 & 17.479180 & 15.710121 \\
\hline $\mathrm{H}$ & 20.776301 & 23.953916 & 17.494041 \\
\hline $\mathrm{H}$ & 16.466332 & 19.823326 & 16.017569 \\
\hline $\mathrm{H}$ & 20.924462 & 14.145569 & 15.287278 \\
\hline $\mathrm{H}$ & 22.358304 & 16.303846 & 13.523564 \\
\hline $\mathrm{H}$ & 16.071461 & 16.682892 & 15.912919 \\
\hline $\mathrm{H}$ & 23.945555 & 20.757212 & 16.402725 \\
\hline $\mathrm{H}$ & 19.325122 & 18.953840 & 16.018448 \\
\hline $\mathrm{H}$ & 18.741271 & 20.747533 & 13.647727 \\
\hline $\mathrm{H}$ & 21.871876 & 24.921134 & 15.112983 \\
\hline $\mathrm{H}$ & 19.090017 & 25.814299 & 14.402290 \\
\hline $\mathrm{H}$ & 19.033801 & 23.401281 & 15.495709 \\
\hline $\mathrm{H}$ & 16.414125 & 22.791431 & 15.527081 \\
\hline $\mathrm{H}$ & 17.555076 & 23.387806 & 13.139148 \\
\hline $\mathrm{H}$ & 23.966950 & 23.294755 & 15.096826 \\
\hline $\mathrm{H}$ & 15.857679 & 20.995494 & 13.410042 \\
\hline $\mathrm{H}$ & 17.104762 & 18.509989 & 14.070344 \\
\hline $\mathrm{H}$ & 19.509017 & 16.712257 & 14.100923 \\
\hline $\mathrm{H}$ & 19.356468 & 16.608130 & 18.242299 \\
\hline $\mathrm{H}$ & 14.186903 & 19.032873 & 15.103085 \\
\hline $\mathrm{H}$ & 10.423676 & 15.600796 & 18.331851 \\
\hline
\end{tabular}




\begin{tabular}{|c|c|c|c|}
\hline $\mathrm{H}$ & 10.205357 & 14.809698 & 16.925298 \\
\hline $\mathrm{H}$ & 11.205340 & 25.079138 & 9.916645 \\
\hline $\mathrm{H}$ & 12.263463 & 26.294819 & 9.667548 \\
\hline $\mathrm{H}$ & 7.982852 & 24.833796 & 16.430095 \\
\hline $\mathrm{H}$ & 8.148067 & 25.412804 & 14.916812 \\
\hline $\mathrm{H}$ & 10.014080 & 29.420622 & 14.694353 \\
\hline $\mathrm{H}$ & 10.112432 & 29.653436 & 16.301395 \\
\hline $\mathrm{H}$ & 11.224723 & 28.213458 & 20.226595 \\
\hline $\mathrm{H}$ & 10.271088 & 26.907887 & 20.425166 \\
\hline $\mathrm{H}$ & 30.691270 & 17.872697 & 17.779770 \\
\hline $\mathrm{H}$ & 31.202567 & 16.514499 & 18.831361 \\
\hline $\mathrm{H}$ & 29.960434 & 17.675174 & 19.395855 \\
\hline $\mathrm{H}$ & 29.118284 & 14.377068 & 12.462132 \\
\hline $\mathrm{H}$ & 28.220161 & 12.827025 & 12.525246 \\
\hline $\mathrm{H}$ & 29.872639 & 12.928689 & 13.211235 \\
\hline $\mathrm{H}$ & 27.091035 & 11.099074 & 19.442898 \\
\hline $\mathrm{H}$ & 25.646278 & 10.951365 & 18.393264 \\
\hline $\mathrm{H}$ & 25.619595 & 12.059874 & 19.792989 \\
\hline $\mathrm{H}$ & 16.891370 & 7.650077 & 17.476792 \\
\hline $\mathrm{H}$ & 18.110329 & 8.070305 & 16.238873 \\
\hline $\mathrm{H}$ & 16.580896 & 8.993079 & 16.340755 \\
\hline $\mathrm{H}$ & 13.754173 & 10.125317 & 20.202709 \\
\hline $\mathrm{H}$ & 13.537490 & 10.737698 & 18.536432 \\
\hline $\mathrm{H}$ & 12.763244 & 11.577964 & 19.912816 \\
\hline $\mathrm{H}$ & 27.221107 & 24.359857 & 11.194170 \\
\hline $\mathrm{H}$ & 28.852775 & 24.049867 & 11.863234 \\
\hline $\mathrm{H}$ & 27.933542 & 25.509471 & 12.361109 \\
\hline $\mathrm{H}$ & 23.228695 & 24.205936 & 9.589976 \\
\hline $\mathrm{H}$ & 24.910162 & 24.178574 & 10.187678 \\
\hline $\mathrm{H}$ & 23.989466 & 25.709786 & 10.186996 \\
\hline $\mathrm{H}$ & 20.639491 & 22.175688 & 9.051223 \\
\hline $\mathrm{H}$ & 20.580939 & 20.387135 & 8.977760 \\
\hline $\mathrm{H}$ & 22.157242 & 21.224024 & 9.095670 \\
\hline $\mathrm{H}$ & 27.406903 & 23.873755 & 19.012891 \\
\hline $\mathrm{H}$ & 28.283736 & 22.309077 & 18.931615 \\
\hline $\mathrm{H}$ & 26.556046 & 22.351920 & 19.405171 \\
\hline $\mathrm{H}$ & 28.512149 & 19.723908 & 12.846806 \\
\hline $\mathrm{H}$ & 29.706671 & 18.682575 & 13.690852 \\
\hline $\mathrm{H}$ & 28.869243 & 20.005845 & 14.574858 \\
\hline $\mathrm{H}$ & 26.269902 & 17.619078 & 19.635258 \\
\hline $\mathrm{H}$ & 25.134848 & 19.009415 & 19.662051 \\
\hline
\end{tabular}




\begin{tabular}{|c|c|c|c|}
\hline $\mathrm{H}$ & 26.902421 & 19.295506 & 19.659185 \\
\hline $\mathrm{H}$ & 23.427883 & 14.270962 & 20.194637 \\
\hline $\mathrm{H}$ & 22.377988 & 15.709817 & 20.008480 \\
\hline $\mathrm{H}$ & 24.162812 & 15.896677 & 20.025110 \\
\hline $\mathrm{H}$ & 24.641395 & 11.226122 & 14.800243 \\
\hline $\mathrm{H}$ & 23.164896 & 11.613437 & 13.855707 \\
\hline $\mathrm{H}$ & 23.088612 & 11.561201 & 15.642185 \\
\hline $\mathrm{H}$ & 22.589814 & 19.938590 & 19.847732 \\
\hline $\mathrm{H}$ & 22.642224 & 18.147614 & 20.001067 \\
\hline $\mathrm{H}$ & 21.074895 & 19.012097 & 20.088826 \\
\hline $\mathrm{H}$ & 20.435808 & 12.745661 & 10.361562 \\
\hline $\mathrm{H}$ & 19.772058 & 14.223047 & 9.602341 \\
\hline $\mathrm{H}$ & 18.729816 & 13.209750 & 10.653921 \\
\hline $\mathrm{H}$ & 20.573989 & 13.734206 & 20.360033 \\
\hline $\mathrm{H}$ & 20.912972 & 12.007145 & 19.991204 \\
\hline $\mathrm{H}$ & 19.242525 & 12.532329 & 20.358971 \\
\hline $\mathrm{H}$ & 17.149714 & 16.398823 & 20.813269 \\
\hline $\mathrm{H}$ & 16.857730 & 14.631770 & 20.966471 \\
\hline $\mathrm{H}$ & 15.472634 & 15.755168 & 20.824891 \\
\hline $\mathrm{H}$ & 15.136183 & 11.312575 & 15.317803 \\
\hline $\mathrm{H}$ & 14.934048 & 12.392773 & 13.898030 \\
\hline $\mathrm{H}$ & 13.663253 & 12.315808 & 15.158919 \\
\hline $\mathrm{H}$ & 18.671008 & 10.012745 & 13.249982 \\
\hline $\mathrm{H}$ & 17.556201 & 11.382301 & 12.942401 \\
\hline $\mathrm{H}$ & 17.232039 & 10.243330 & 14.287374 \\
\hline $\mathrm{H}$ & 27.993575 & 18.060473 & 9.089143 \\
\hline $\mathrm{H}$ & 28.631573 & 17.510087 & 10.673341 \\
\hline $\mathrm{H}$ & 27.800826 & 19.084684 & 10.547128 \\
\hline $\mathrm{H}$ & 24.678668 & 21.234461 & 8.900135 \\
\hline $\mathrm{H}$ & 26.259026 & 20.498733 & 9.285963 \\
\hline $\mathrm{H}$ & 25.971331 & 22.228227 & 9.643659 \\
\hline $\mathrm{H}$ & 22.515823 & 18.582622 & 8.669027 \\
\hline $\mathrm{H}$ & 21.414219 & 17.170836 & 8.791609 \\
\hline $\mathrm{H}$ & 23.186475 & 16.924112 & 8.724110 \\
\hline $\mathrm{H}$ & 24.488849 & 11.892894 & 11.344440 \\
\hline $\mathrm{H}$ & 23.550143 & 12.060671 & 9.823918 \\
\hline $\mathrm{H}$ & 22.730826 & 12.199570 & 11.410882 \\
\hline $\mathrm{H}$ & 28.260189 & 9.539228 & 17.629083 \\
\hline $\mathrm{H}$ & 29.176942 & 8.522141 & 16.477766 \\
\hline $\mathrm{H}$ & 27.645345 & 9.300134 & 15.982714 \\
\hline $\mathrm{H}$ & 27.282739 & 14.931738 & 7.957681 \\
\hline
\end{tabular}




\begin{tabular}{|c|c|c|c|}
\hline $\mathrm{H}$ & 26.199230 & 13.535335 & 8.083954 \\
\hline $\mathrm{H}$ & 27.943457 & 13.277337 & 7.772846 \\
\hline $\mathrm{H}$ & 31.269694 & 16.715933 & 13.634478 \\
\hline $\mathrm{H}$ & 32.954674 & 16.718493 & 14.235412 \\
\hline $\mathrm{H}$ & 31.648918 & 17.493740 & 15.181810 \\
\hline $\mathrm{H}$ & 28.175764 & 26.334410 & 17.468944 \\
\hline $\mathrm{H}$ & 28.128230 & 27.007701 & 15.835228 \\
\hline $\mathrm{H}$ & 29.599479 & 27.217732 & 16.834601 \\
\hline $\mathrm{H}$ & 27.389701 & 13.912963 & 21.766122 \\
\hline $\mathrm{H}$ & 28.315649 & 15.426418 & 21.733161 \\
\hline $\mathrm{H}$ & 28.824994 & 14.097712 & 22.817057 \\
\hline $\mathrm{H}$ & 18.956041 & 16.838062 & 9.505653 \\
\hline $\mathrm{H}$ & 18.996214 & 18.594664 & 9.156251 \\
\hline $\mathrm{H}$ & 17.437054 & 17.787995 & 9.498409 \\
\hline $\mathrm{H}$ & 15.887257 & 13.508668 & 11.431527 \\
\hline $\mathrm{H}$ & 16.226511 & 15.039973 & 10.571163 \\
\hline $\mathrm{H}$ & 14.670284 & 14.814616 & 11.420142 \\
\hline $\mathrm{H}$ & 24.016795 & 24.265501 & 19.916980 \\
\hline $\mathrm{H}$ & 23.003467 & 25.743010 & 19.903150 \\
\hline $\mathrm{H}$ & 24.772104 & 25.887514 & 19.670966 \\
\hline $\mathrm{H}$ & 24.969627 & 27.181556 & 12.309006 \\
\hline $\mathrm{H}$ & 26.311552 & 27.480455 & 13.456551 \\
\hline $\mathrm{H}$ & 24.848522 & 28.509147 & 13.512675 \\
\hline $\mathrm{H}$ & 17.791875 & 20.821045 & 19.957841 \\
\hline $\mathrm{H}$ & 17.769882 & 22.617694 & 19.878262 \\
\hline $\mathrm{H}$ & 19.332204 & 21.738314 & 19.922310 \\
\hline $\mathrm{H}$ & 19.186407 & 26.309186 & 9.287177 \\
\hline $\mathrm{H}$ & 19.795649 & 24.719408 & 8.740148 \\
\hline $\mathrm{H}$ & 20.909292 & 25.882716 & 9.532462 \\
\hline $\mathrm{H}$ & 16.994463 & 20.373358 & 8.713917 \\
\hline $\mathrm{H}$ & 18.070739 & 21.798973 & 8.539234 \\
\hline $\mathrm{H}$ & 16.294291 & 22.016740 & 8.594276 \\
\hline $\mathrm{H}$ & 12.939342 & 16.993334 & 20.127127 \\
\hline $\mathrm{H}$ & 12.093508 & 18.545214 & 19.812932 \\
\hline $\mathrm{H}$ & 13.853155 & 18.530315 & 20.157756 \\
\hline $\mathrm{H}$ & 11.938505 & 14.133742 & 13.906368 \\
\hline $\mathrm{H}$ & 12.425537 & 15.120531 & 12.498070 \\
\hline $\mathrm{H}$ & 10.887389 & 15.474544 & 13.339664 \\
\hline $\mathrm{H}$ & 16.785502 & 28.074233 & 12.737705 \\
\hline $\mathrm{H}$ & 16.952707 & 28.446178 & 14.479582 \\
\hline $\mathrm{H}$ & 15.362374 & 28.646281 & 13.669400 \\
\hline
\end{tabular}




\begin{tabular}{|c|c|c|c|}
\hline $\mathrm{H}$ & 19.810093 & 27.268078 & 19.221192 \\
\hline $\mathrm{H}$ & 19.454639 & 28.880594 & 18.510209 \\
\hline $\mathrm{H}$ & 21.144562 & 28.415950 & 18.871273 \\
\hline $\mathrm{H}$ & 21.202280 & 29.474997 & 11.428411 \\
\hline $\mathrm{H}$ & 22.224271 & 28.011968 & 11.260654 \\
\hline $\mathrm{H}$ & 22.730997 & 29.341591 & 12.349649 \\
\hline $\mathrm{H}$ & 16.985124 & 26.684581 & 19.444836 \\
\hline $\mathrm{H}$ & 18.029571 & 25.227413 & 19.447763 \\
\hline $\mathrm{H}$ & 16.255023 & 25.059948 & 19.643892 \\
\hline $\mathrm{H}$ & 11.098583 & 20.010526 & 15.304684 \\
\hline $\mathrm{H}$ & 11.297178 & 20.002089 & 13.529013 \\
\hline $\mathrm{H}$ & 10.177931 & 21.178354 & 14.292819 \\
\hline $\mathrm{H}$ & 14.047853 & 16.844507 & 10.355997 \\
\hline $\mathrm{H}$ & 14.793698 & 18.168304 & 9.406293 \\
\hline $\mathrm{H}$ & 13.141649 & 18.360095 & 10.058499 \\
\hline $\mathrm{H}$ & 14.503339 & 23.073814 & 19.589246 \\
\hline $\mathrm{H}$ & 15.230521 & 21.442868 & 19.765209 \\
\hline $\mathrm{H}$ & 13.457444 & 21.658236 & 19.923003 \\
\hline $\mathrm{H}$ & 26.651480 & 29.600168 & 16.540171 \\
\hline $\mathrm{H}$ & 27.452399 & 28.938084 & 17.998946 \\
\hline $\mathrm{H}$ & 26.552939 & 30.476129 & 18.098098 \\
\hline $\mathrm{H}$ & 16.075254 & 26.958429 & 8.903570 \\
\hline $\mathrm{H}$ & 16.998110 & 27.059365 & 10.435264 \\
\hline $\mathrm{H}$ & 15.245884 & 27.406110 & 10.429459 \\
\hline $\mathrm{H}$ & 11.901703 & 20.221226 & 11.102417 \\
\hline $\mathrm{H}$ & 11.584885 & 20.976051 & 9.508325 \\
\hline $\mathrm{H}$ & 11.047282 & 21.788104 & 11.014655 \\
\hline $\mathrm{H}$ & 11.944354 & 13.216740 & 17.455080 \\
\hline $\mathrm{H}$ & 10.673783 & 13.217024 & 18.716928 \\
\hline $\mathrm{H}$ & 12.223296 & 14.084019 & 18.970348 \\
\hline $\mathrm{H}$ & 10.639418 & 25.190093 & 12.341555 \\
\hline $\mathrm{H}$ & 11.314321 & 26.832347 & 12.076795 \\
\hline $\mathrm{H}$ & 9.773469 & 26.635282 & 12.965572 \\
\hline $\mathrm{H}$ & 14.607577 & 29.597123 & 17.246262 \\
\hline $\mathrm{H}$ & 14.706943 & 28.742649 & 18.811071 \\
\hline $\mathrm{H}$ & 13.218965 & 29.641866 & 18.376843 \\
\hline $\mathrm{H}$ & 21.987742 & 31.925600 & 13.906787 \\
\hline $\mathrm{H}$ & 23.517004 & 31.018227 & 14.107723 \\
\hline $\mathrm{H}$ & 23.275391 & 32.563073 & 14.971901 \\
\hline $\mathrm{H}$ & 9.614305 & 22.669624 & 18.276186 \\
\hline $\mathrm{H}$ & 9.183998 & 24.201874 & 19.100504 \\
\hline
\end{tabular}




\begin{tabular}{|c|c|c|c|}
\hline $\mathrm{H}$ & 10.444484 & 23.131548 & 19.786882 \\
\hline $\mathrm{H}$ & 11.968736 & 23.918175 & 7.929561 \\
\hline $\mathrm{H}$ & 13.116515 & 25.241586 & 7.662728 \\
\hline $\mathrm{H}$ & 11.358569 & 25.551763 & 7.520927 \\
\hline $\mathrm{H}$ & 8.499950 & 22.582276 & 15.698925 \\
\hline $\mathrm{H}$ & 8.676963 & 23.216255 & 14.053014 \\
\hline $\mathrm{H}$ & 7.069540 & 23.224291 & 14.838048 \\
\hline $\mathrm{H}$ & 12.052106 & 30.662703 & 14.306600 \\
\hline $\mathrm{H}$ & 12.175471 & 30.908887 & 16.059223 \\
\hline $\mathrm{H}$ & 10.835500 & 31.667807 & 15.147304 \\
\hline $\mathrm{H}$ & 13.111713 & 27.081064 & 21.237070 \\
\hline $\mathrm{H}$ & 12.072701 & 25.659152 & 21.454607 \\
\hline $\mathrm{H}$ & 11.726036 & 27.160367 & 22.364383 \\
\hline $\mathrm{H}$ & 17.223857 & 18.723462 & 18.282953 \\
\hline $\mathrm{H}$ & 22.609409 & 21.821429 & 18.083811 \\
\hline $\mathrm{H}$ & 13.770676 & 24.676058 & 16.156067 \\
\hline $\mathrm{H}$ & 26.346243 & 15.584182 & 16.421007 \\
\hline $\mathrm{C}$ & 30.355227 & 17.140241 & 18.522990 \\
\hline $\mathrm{C}$ & 28.931512 & 13.472693 & 13.056495 \\
\hline $\mathrm{C}$ & 26.248114 & 11.641685 & 18.996440 \\
\hline $\mathrm{C}$ & 17.352262 & 8.474993 & 16.920074 \\
\hline $\mathrm{C}$ & 13.654374 & 11.026461 & 19.586145 \\
\hline $\mathrm{C}$ & 27.855661 & 24.451758 & 12.082838 \\
\hline $\mathrm{C}$ & 23.918676 & 24.623233 & 10.332561 \\
\hline $\mathrm{C}$ & 21.105352 & 21.248074 & 9.406869 \\
\hline $\mathrm{C}$ & 27.320706 & 22.808429 & 18.766570 \\
\hline $\mathrm{C}$ & 28.766588 & 19.238910 & 13.796659 \\
\hline $\mathrm{C}$ & 26.098445 & 18.646835 & 19.288684 \\
\hline $\mathrm{C}$ & 23.330312 & 15.253068 & 19.715858 \\
\hline $\mathrm{C}$ & 23.716642 & 11.817811 & 14.780074 \\
\hline $\mathrm{C}$ & 22.068085 & 19.000340 & 19.623580 \\
\hline $\mathrm{C}$ & 19.746714 & 13.587746 & 10.496727 \\
\hline $\mathrm{C}$ & 20.188568 & 12.824257 & 19.886519 \\
\hline $\mathrm{C}$ & 16.505676 & 15.565075 & 20.511070 \\
\hline $\mathrm{C}$ & 14.743954 & 12.276368 & 14.972541 \\
\hline $\mathrm{C}$ & 18.012763 & 10.756053 & 13.716484 \\
\hline $\mathrm{C}$ & 27.820563 & 18.053007 & 10.173592 \\
\hline $\mathrm{C}$ & 25.513592 & 21.233860 & 9.611608 \\
\hline $\mathrm{C}$ & 22.386768 & 17.579160 & 9.092003 \\
\hline $\mathrm{C}$ & 23.654697 & 12.412885 & 10.858727 \\
\hline $\mathrm{C}$ & 28.556165 & 9.425016 & 16.579859 \\
\hline
\end{tabular}




\begin{tabular}{|c|c|c|c|}
\hline $\mathrm{C}$ & 27.209121 & 13.896408 & 8.310294 \\
\hline $\mathrm{C}$ & 31.896495 & 16.646170 & 14.531592 \\
\hline $\mathrm{C}$ & 28.780157 & 26.529443 & 16.575622 \\
\hline $\mathrm{C}$ & 28.398457 & 14.337586 & 21.831629 \\
\hline $\mathrm{C}$ & 18.506321 & 17.810263 & 9.743416 \\
\hline $\mathrm{C}$ & 15.744998 & 14.597817 & 11.450816 \\
\hline $\mathrm{C}$ & 23.891644 & 25.261394 & 19.478477 \\
\hline $\mathrm{C}$ & 25.221445 & 27.494715 & 13.330440 \\
\hline $\mathrm{C}$ & 18.296483 & 21.710784 & 19.561562 \\
\hline $\mathrm{C}$ & 19.884433 & 25.494202 & 9.512147 \\
\hline $\mathrm{C}$ & 17.136813 & 21.428215 & 8.978496 \\
\hline $\mathrm{C}$ & 13.030104 & 17.989343 & 19.676782 \\
\hline $\mathrm{C}$ & 11.924009 & 15.140613 & 13.471924 \\
\hline $\mathrm{C}$ & 16.280669 & 28.047030 & 13.709722 \\
\hline $\mathrm{C}$ & 20.166579 & 28.046820 & 18.537777 \\
\hline $\mathrm{C}$ & 21.875226 & 28.785407 & 11.953092 \\
\hline $\mathrm{C}$ & 17.053721 & 25.629223 & 19.152105 \\
\hline $\mathrm{C}$ & 11.126625 & 20.638815 & 14.405444 \\
\hline $\mathrm{C}$ & 14.132824 & 17.931031 & 10.249361 \\
\hline $\mathrm{C}$ & 14.361160 & 22.001872 & 19.404003 \\
\hline $\mathrm{C}$ & 26.576862 & 29.486417 & 17.626781 \\
\hline $\mathrm{C}$ & 16.034334 & 26.786264 & 9.987222 \\
\hline $\mathrm{C}$ & 11.832266 & 21.172452 & 10.559905 \\
\hline $\mathrm{C}$ & 11.457798 & 13.818701 & 18.231239 \\
\hline $\mathrm{C}$ & 10.759564 & 26.191903 & 12.774813 \\
\hline $\mathrm{C}$ & 14.038357 & 29.024187 & 17.988055 \\
\hline $\mathrm{C}$ & 22.780602 & 31.651847 & 14.613770 \\
\hline $\mathrm{C}$ & 10.003884 & 23.513882 & 18.857229 \\
\hline $\mathrm{C}$ & 12.130389 & 24.993349 & 8.072912 \\
\hline $\mathrm{C}$ & 8.149769 & 23.355669 & 15.004542 \\
\hline $\mathrm{C}$ & 11.471728 & 30.773534 & 15.230138 \\
\hline $\mathrm{C}$ & 12.078664 & 26.754416 & 21.404351 \\
\hline $\mathrm{N}$ & 29.219218 & 13.810842 & 20.709350 \\
\hline $\mathrm{N}$ & 27.408943 & 13.874808 & 9.781407 \\
\hline $\mathrm{N}$ & 31.581001 & 15.382183 & 15.241877 \\
\hline $\mathrm{N}$ & 29.261380 & 10.657730 & 16.137992 \\
\hline $\mathrm{N}$ & 29.275149 & 25.238420 & 16.029061 \\
\hline $\mathrm{N}$ & 10.917290 & 15.060828 & 17.615972 \\
\hline $\mathrm{N}$ & 12.126825 & 25.291134 & 9.527591 \\
\hline $\mathrm{N}$ & 8.491936 & 24.690505 & 15.554099 \\
\hline $\mathrm{N}$ & 10.683225 & 29.533292 & 15.459988 \\
\hline
\end{tabular}




\begin{tabular}{|c|c|c|c|}
\hline $\mathrm{N}$ & 11.239981 & 27.190837 & 20.256989 \\
\hline $\mathrm{S}$ & 20.259062 & 14.572764 & 11.955527 \\
\hline S & 22.499339 & 17.652213 & 10.913142 \\
\hline $\mathrm{S}$ & 23.931889 & 14.227906 & 10.852616 \\
\hline $\mathrm{S}$ & 27.416435 & 18.079941 & 14.263987 \\
\hline $\mathrm{S}$ & 18.705485 & 18.139212 & 11.531110 \\
\hline $\mathrm{S}$ & 21.017940 & 21.199279 & 11.232698 \\
\hline S & 24.152524 & 13.599917 & 14.864913 \\
\hline $\mathrm{S}$ & 26.128473 & 18.657153 & 17.458231 \\
\hline $\mathrm{S}$ & 21.854772 & 18.874315 & 17.811302 \\
\hline S & 26.200663 & 17.263799 & 10.527615 \\
\hline $\mathrm{S}$ & 24.902889 & 20.756880 & 11.274072 \\
\hline S & 16.543022 & 15.365816 & 18.689377 \\
\hline $\mathrm{S}$ & 29.026672 & 16.100375 & 17.796245 \\
\hline S & 27.105931 & 23.504507 & 13.462942 \\
\hline $\mathrm{S}$ & 23.320028 & 24.279417 & 12.032645 \\
\hline $\mathrm{S}$ & 19.886193 & 13.132214 & 18.107238 \\
\hline $\mathrm{S}$ & 23.376169 & 15.028913 & 17.898916 \\
\hline $\mathrm{S}$ & 16.463582 & 15.274937 & 12.997195 \\
\hline S & 26.829548 & 22.619382 & 17.010610 \\
\hline $\mathrm{S}$ & 26.857192 & 13.004802 & 17.927371 \\
\hline S & 19.023862 & 11.793081 & 14.841949 \\
\hline $\mathrm{S}$ & 28.191228 & 13.964534 & 14.660671 \\
\hline $\mathrm{S}$ & 15.567514 & 13.633829 & 15.876096 \\
\hline $\mathrm{S}$ & 23.681536 & 25.124413 & 17.662249 \\
\hline S & 24.449044 & 26.336020 & 14.512759 \\
\hline $\mathrm{S}$ & 15.127551 & 12.101754 & 19.849107 \\
\hline $\mathrm{S}$ & 18.185839 & 9.593005 & 18.120120 \\
\hline $\mathrm{S}$ & 18.326331 & 21.590677 & 17.737513 \\
\hline $\mathrm{S}$ & 19.473076 & 24.772558 & 11.146187 \\
\hline $\mathrm{S}$ & 17.180649 & 21.617777 & 10.793282 \\
\hline $\mathrm{S}$ & 13.379178 & 17.826318 & 17.883330 \\
\hline $\mathrm{S}$ & 12.804645 & 16.306248 & 14.580859 \\
\hline $\mathrm{S}$ & 15.840926 & 26.316264 & 14.137294 \\
\hline $\mathrm{S}$ & 20.339858 & 27.364447 & 16.847382 \\
\hline S & 20.947752 & 28.011905 & 13.332709 \\
\hline $\mathrm{S}$ & 16.856863 & 25.504396 & 17.335780 \\
\hline S & 12.512406 & 21.840309 & 14.552338 \\
\hline $\mathrm{S}$ & 14.828451 & 18.657164 & 11.783157 \\
\hline S & 14.134278 & 21.739785 & 17.603597 \\
\hline S & 25.064024 & 28.558483 & 18.120192 \\
\hline
\end{tabular}




\begin{tabular}{|c|c|c|c|}
\hline S & 15.715067 & 25.005442 & 10.299226 \\
\hline S & 13.456793 & 22.022513 & 10.652293 \\
\hline S & 11.714742 & 26.041048 & 14.332629 \\
\hline S & 13.371216 & 27.507557 & 17.197743 \\
\hline S & 22.008695 & 30.804691 & 16.050563 \\
\hline S & 11.300890 & 24.379834 & 17.885424 \\
\hline $\mathrm{Cu}$ & 21.507785 & 22.026593 & 14.496334 \\
\hline $\mathrm{Cu}$ & 19.954312 & 19.958043 & 14.690198 \\
\hline $\mathrm{Cu}$ & 20.287248 & 24.432596 & 14.676203 \\
\hline $\mathrm{Cu}$ & 22.708193 & 19.521731 & 14.063440 \\
\hline $\mathrm{Cu}$ & 18.777817 & 22.424572 & 14.016394 \\
\hline $\mathrm{Cu}$ & 24.099291 & 21.583746 & 14.852879 \\
\hline $\mathrm{Cu}$ & 20.111082 & 22.622420 & 16.640547 \\
\hline $\mathrm{Cu}$ & 22.200282 & 20.917250 & 16.741282 \\
\hline $\mathrm{Cu}$ & 22.347703 & 23.267016 & 17.463464 \\
\hline $\mathrm{Cu}$ & 23.189166 & 21.982193 & 12.215937 \\
\hline $\mathrm{Cu}$ & 21.127056 & 23.530190 & 12.133450 \\
\hline $\mathrm{Cu}$ & 17.211117 & 20.266249 & 14.331084 \\
\hline $\mathrm{Cu}$ & 18.841482 & 20.486991 & 11.875940 \\
\hline $\mathrm{Cu}$ & 20.913156 & 18.930278 & 11.946887 \\
\hline $\mathrm{Cu}$ & 21.187956 & 17.377390 & 14.346953 \\
\hline $\mathrm{Cu}$ & 22.316016 & 15.564543 & 11.863991 \\
\hline $\mathrm{Cu}$ & 22.289682 & 14.946690 & 14.594076 \\
\hline $\mathrm{Cu}$ & 24.602154 & 14.810685 & 12.952443 \\
\hline $\mathrm{Cu}$ & 19.702497 & 15.534616 & 15.391953 \\
\hline $\mathrm{Cu}$ & 20.013917 & 17.646007 & 17.049293 \\
\hline $\mathrm{Cu}$ & 17.781860 & 17.246978 & 18.041031 \\
\hline $\mathrm{Cu}$ & 18.726237 & 15.028963 & 17.706162 \\
\hline $\mathrm{Cu}$ & 18.660905 & 15.970693 & 12.818841 \\
\hline $\mathrm{Cu}$ & 20.013342 & 13.526021 & 13.878276 \\
\hline $\mathrm{Cu}$ & 17.836654 & 13.600552 & 15.544761 \\
\hline $\mathrm{Cu}$ & 21.401866 & 14.388909 & 17.020738 \\
\hline $\mathrm{Cu}$ & 23.929564 & 17.031820 & 14.030689 \\
\hline $\mathrm{Cu}$ & 25.403358 & 19.270199 & 14.157276 \\
\hline $\mathrm{Cu}$ & 23.901413 & 19.032643 & 16.484187 \\
\hline $\mathrm{Cu}$ & 25.092072 & 16.750388 & 16.410254 \\
\hline $\mathrm{Cu}$ & 26.145227 & 16.860537 & 12.766011 \\
\hline $\mathrm{Cu}$ & 24.515238 & 18.529315 & 11.560894 \\
\hline $\mathrm{Cu}$ & 25.901309 & 21.684853 & 13.008763 \\
\hline $\mathrm{Cu}$ & 25.837109 & 20.674072 & 16.449401 \\
\hline $\mathrm{Cu}$ & 22.504673 & 16.824152 & 16.786355 \\
\hline
\end{tabular}




\begin{tabular}{|c|c|c|c|}
\hline $\mathrm{Cu}$ & 24.806166 & 23.466355 & 16.551843 \\
\hline $\mathrm{Cu}$ & 24.903308 & 24.082953 & 13.909278 \\
\hline $\mathrm{Cu}$ & 17.910481 & 19.427113 & 16.929616 \\
\hline $\mathrm{Cu}$ & 15.337146 & 16.860879 & 17.425570 \\
\hline $\mathrm{Cu}$ & 27.627934 & 17.104729 & 16.316427 \\
\hline $\mathrm{Cu}$ & 26.282196 & 15.324536 & 14.697616 \\
\hline $\mathrm{Cu}$ & 15.583807 & 13.305084 & 18.057344 \\
\hline $\mathrm{Cu}$ & 25.246499 & 14.193337 & 16.849585 \\
\hline $\mathrm{Cu}$ & 15.017111 & 15.734984 & 14.946667 \\
\hline $\mathrm{Cu}$ & 28.436902 & 14.246742 & 18.842786 \\
\hline $\mathrm{Cu}$ & 28.222178 & 12.433697 & 16.268014 \\
\hline $\mathrm{Cu}$ & 26.022060 & 15.052315 & 10.785824 \\
\hline $\mathrm{Cu}$ & 29.589027 & 15.146786 & 15.888661 \\
\hline $\mathrm{Cu}$ & 27.829002 & 23.893425 & 15.499844 \\
\hline $\mathrm{Cu}$ & 16.680307 & 10.954919 & 18.855424 \\
\hline $\mathrm{Cu}$ & 18.823717 & 11.438419 & 17.076353 \\
\hline $\mathrm{Cu}$ & 18.468064 & 17.830415 & 14.913285 \\
\hline $\mathrm{Cu}$ & 17.677502 & 24.907400 & 13.972421 \\
\hline $\mathrm{Cu}$ & 21.457260 & 25.409613 & 16.734531 \\
\hline $\mathrm{Cu}$ & 19.890601 & 26.129599 & 12.827226 \\
\hline $\mathrm{Cu}$ & 18.730487 & 25.951202 & 16.169664 \\
\hline $\mathrm{Cu}$ & 16.023625 & 22.729706 & 13.806395 \\
\hline $\mathrm{Cu}$ & 17.657359 & 23.552938 & 16.509901 \\
\hline $\mathrm{Cu}$ & 14.495883 & 20.609955 & 14.411686 \\
\hline $\mathrm{Cu}$ & 15.878699 & 18.270696 & 15.318377 \\
\hline $\mathrm{Cu}$ & 16.610923 & 17.560740 & 12.749378 \\
\hline $\mathrm{Cu}$ & 16.214849 & 21.389338 & 16.618007 \\
\hline $\mathrm{Cu}$ & 22.167633 & 26.344618 & 14.291289 \\
\hline $\mathrm{Cu}$ & 24.523271 & 27.070902 & 16.584295 \\
\hline $\mathrm{Cu}$ & 17.395685 & 23.826854 & 11.411861 \\
\hline $\mathrm{Cu}$ & 15.217367 & 24.807775 & 12.518180 \\
\hline $\mathrm{Cu}$ & 13.673533 & 22.784479 & 12.797335 \\
\hline $\mathrm{Cu}$ & 15.013866 & 23.534914 & 16.082000 \\
\hline $\mathrm{Cu}$ & 13.927011 & 18.005165 & 13.684541 \\
\hline $\mathrm{Cu}$ & 14.291653 & 19.607869 & 16.845385 \\
\hline $\mathrm{Cu}$ & 15.208744 & 20.921156 & 11.744898 \\
\hline $\mathrm{Cu}$ & 12.292832 & 16.283073 & 16.722544 \\
\hline $\mathrm{Cu}$ & 13.599795 & 24.703002 & 14.392235 \\
\hline $\mathrm{Cu}$ & 14.905025 & 26.111854 & 16.272092 \\
\hline $\mathrm{Cu}$ & 21.319519 & 28.799042 & 15.422066 \\
\hline $\mathrm{Cu}$ & 12.513568 & 23.159447 & 16.407153 \\
\hline
\end{tabular}




$\begin{array}{llll}\mathrm{Cu} & 23.521668 & 29.564541 & 16.967696 \\ \mathrm{Cu} & 13.609324 & 24.249554 & 10.550651 \\ \mathrm{Cu} & 10.548240 & 25.053030 & 15.922456 \\ \mathrm{Cu} & 11.771826 & 27.801203 & 15.677341 \\ \mathrm{Cu} & 11.888046 & 26.439965 & 18.433694\end{array}$

